\section{(C) OPEN ACCESS}

\title{
Sex differences in risk factor management of coronary heart disease across three regions
}

\author{
Min Zhao, ${ }_{1}^{1}$ Ilonca Vaartjes, ${ }^{2,3}$ Ian Graham, ${ }^{4}$ Diederick Grobbee, ${ }^{1,3}$ Wilko Spiering, ${ }^{5}$ \\ Kerstin Klipstein-Grobusch, ${ }^{1,6}$ Mark Woodward, ${ }^{7,8}$ Sanne AE Peters ${ }^{7}$
}

- Additional material is published online only. To view please visit the journal online (http://dx.doi.org/10.1136/ heartjnl-2017-311429)

For numbered affiliations see end of article.

\section{Correspondence to}

Min Zhao, Julius Global Health, Julius Center for Health Sciences and Primary Care, University Medical Center Utrecht, Utrecht, The Netherlands; m.zhao@ umcutrecht.nl, minzhao0208@ gmail.com

Received 24 February 2017 Revised 22 May 2017

Accepted 19 June 2017

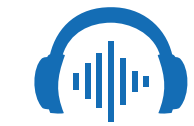

Listen to Podcast www.goo.gl/U6bq7i

\section{Linked}

- http://dx.doi.org/10.1136/ heartjinl-2017-311921

\section{CrossMark}

To cite: Zhao M, Vaartjes I, Graham I, et al. Heart 2017;103:1587-1594.

\section{ABSTRACT}

Objective To investigate whether there are sex differences in risk factor management of patients with established coronary heart disease (CHD), and to assess demographic variations of any potential sex differences. Methods Patients with CHD were recruited from Europe, Asia, and the Middle East between 2012-2013. Adherence to guideline-recommended treatment and lifestyle targets was assessed and summarised as a Cardiovascular Health Index Score (CHIS). Age-adjusted regression models were used to estimate odds ratios for women versus men in risk factor management.

Results 10112 patients (29\% women) were included. Compared with men, women were less likely to achieve targets for total cholesterol (OR 0.50, 95\% Cl 0.43 to 0.59), low-density lipoprotein cholesterol (OR 0.57, 95\% $\mathrm{Cl} 0.51$ to 0.64 ), and glucose (OR $0.78,95 \% \mathrm{Cl} 0.70$ to 0.87 ), or to be physically active (OR $0.74,95 \% \mathrm{Cl} 0.68$ to 0.81 ) or non-obese (OR $0.82,95 \% \mathrm{Cl} 0.74$ to 0.90 ). In contrast, women had better control of blood pressure (OR 1.31, 95\% Cl 1.20 to 1.44) and were more likely to be a non-smoker (OR 1.93, 95\% Cl 1.67 to 2.22) than men. Overall, women were less likely than men to achieve all treatment targets (OR 0.75, 95\% Cl 0.60 to 0.93 ) or obtain an adequate CHIS (OR $0.81,95 \% \mathrm{CI}$ 0.73 to 0.91 ), but no significant differences were found for all lifestyle targets (OR $0.93,95 \% \mathrm{Cl} 0.84$ to 1.02). Sex disparities in reaching treatment targets were smaller in Europe than in Asia and the Middle East. Women in Asia were more likely than men to reach lifestyle targets, with opposing results in Europe and the Middle East. Conclusions Risk factor management for the secondary prevention of CHD was generally worse in women than in men. The magnitude and direction of the sex differences varied by region.

\section{INTRODUCTION}

Coronary heart disease (CHD) remains one of the leading causes of death and disability worldwide. In 2015, 16\% of all deaths in both men and women were caused by CHD. ${ }^{1}$ Individuals with established CHD are at high risk of further events and require intensive risk factor management. ${ }^{2}$ Despite convincing evidence on the major benefits of the management of modifiable risk factors in subjects with established CHD, an unacceptably large proportion of affected individuals do not reach guideline recommended risk factor targets. ${ }^{3-5}$

Previous studies in Western populations have suggested that the control of cardiovascular risk factors among patients with established CHD is generally even lower in women than in men. ${ }^{6-8}$ For example, results from EUROASPIRE III indicated that, despite similar treatment rates, women were less likely than men to achieve medical target levels. EUROASPIRE IV largely confirmed these findings and also reported that sex differences were primarily seen among individuals with a lower education level or at older age, suggesting a double burden among women in these populations. Despite the growing burden of CHD in non-Western countries, such as those in Asia and the Middle East, it remains unknown whether sex differences in risk factor control for the secondary prevention of CHD also exist across geographically diverse regions.

We therefore used data from the SUrvey of Risk Factors (SURF) Phase I audit to investigate whether there are sex differences in the management of CHD risk factors among patients with established CHD from three diverse regions.

\section{METHODS}

\section{Study population}

Details of the study protocol and methodology of SURF have been previously reported. ${ }^{45}$ Between 2012 and 2013, consecutive patients aged $\geq 18$ years with established CHD (defined as a history of coronary artery bypass graft surgery (CABG), percutaneous coronary intervention (PCI), acute coronary syndromes (ACS), or stable angina) were recruited from routine outpatient cardiology clinics in 11 countries across three regions: Europe (Belgium, Croatia, Denmark, Ireland, Italy, Northern Ireland, Romania, and Russia); Asia (Taiwan and China); and the Middle East (Saudi Arabia). Data on demographics, self-reported smoking status, physical activity, attendance of cardiac rehabilitation, physical and laboratory measurements (ie, body anthropometry, blood pressure (BP), cholesterol, blood glucose, and glycated haemoglobin (HbA1c)), and prescription of medications were obtained by trained research staff using standardised procedures.

\section{Risk factor targets}

The Joint European Societies guidelines were used to assess whether recommended targets for risk factor management were met. ${ }^{2} 9$ The BP target was $<140 / 90 \mathrm{mmHg}$ for patients without diabetes and $<140 / 80 \mathrm{mmHg}$ for patients with diabetes. The targets for total cholesterol (TC), low-density lipoprotein (LDL) cholesterol, and blood glucose were $<3 \mathrm{mmol} / \mathrm{L},<1.8 \mathrm{mmol} / \mathrm{L}$, and $<7 \mathrm{mmol} / \mathrm{L}$, respectively. HbA1c was only collected for patients 
with diabetes and its target was $<7 \%$. While the guideline does not define targets for high-density lipoprotein (HDL) cholesterol, values $>1.0 \mathrm{mmol} / \mathrm{L}$ for men and $>1.2 \mathrm{mmol} / \mathrm{L}$ for women were regarded as desirable. Obesity was defined as a body mass index $(\mathrm{BMI}) \geq 30 \mathrm{~kg} / \mathrm{m}^{2}$ and central obesity was defined as waist circumference $\geq 88 \mathrm{~cm}$ for women and $\geq 102 \mathrm{~cm}$ for men. ${ }^{10}$ Adequate physical activity was defined as moderate or vigorous physical activity for at least $30 \mathrm{~min}$ three or more times a week.

A Cardiovascular Health Index Score (CHIS), adapted from the ideal Cardiovascular Health Score, ${ }^{11}$ was used to summarise overall risk factor management. Since dietary information was not available, the CHIS included six risk factors: smoking status (current smoker vs non-smoker (never/ex-smoker)), BMI (obese vs not), physical activity (adequate vs not), BP (on target vs not), LDL cholesterol (on target vs not), and HbA1c/glucose (on target vs not). ${ }^{4}$ The number of risk factors on target could range from 0 to 6 and the risk factor profile was considered satisfactory if five or more risk factors were controlled. Additionally, risk factor control was assessed separately for therapeutic and lifestyle targets. 'All treatment targets' was defined as reaching targets for BP, LDL cholesterol, and HbA1c/glucose. 'All lifestyle targets' was defined as reaching targets for smoking status, BMI, and physical activity.

\section{Statistical analyses}

Patient characteristics were presented as means (SD) for continuous variables and as percentages for categorical variables, separately for men and women. Age-adjusted logistic regression analyses were used to obtain odds ratios (OR, 95\% CI) of women versus men for sex associated with individual and combined risk factor targets. Men thus served as the reference group. Complete case analyses were conducted. Stratified analyses were performed by region (Europe, Asia, and the Middle East) and age group ( $\leq 65$ years and $>65$ years). Effect modification was assessed by adding an interaction term to the model. In secondary analyses, we additionally adjusted for BP, smoking status, TC, HDL cholesterol, and glucose.

To assess the impact of medication use on therapeutic target achievements, the analyses on the target achievements of BP, TC and LDL cholesterol, glucose and HbA1c were stratified by the use of antihypertensive, lipid-lowering, and anti-diabetic medications, respectively. We also assessed whether the findings differed between defined CHD category (CABG, PCI, ACS, or stable angina). All analyses were performed with $\mathrm{R}$ version 3.2.2 and all tests were two tailed with statistical significance set at the $5 \%$ level.

\section{RESULTS}

A total of 10112 patients, of whom 2958 (29\%) were women, were included. On average, women were 4 years older than men; more women than men had stable angina but fewer had CABG. Women more frequently had a history of hypertension and diabetes (table 1). Prescriptions of antiplatelet and lipid lowering therapy were less frequent in women than men. The percentage of data not recorded was broadly similar between the sexes (supplementary eTable 1).

\section{Achievement of risk factor targets}

Control of cardiovascular risk factors was suboptimal in both men and women for all risk factors examined (figure 1). BP levels were on target in $45 \%$ of women and $38 \%$ of men. The corresponding age-adjusted OR was 1.31 (95\% CI 1.20 to 1.44), indicating that women had a $31 \%$ higher odds of meeting the BP target than men. Women were also more likely than men to be non-smokers; the OR for being a non-smoker was 1.93 (95\% CI 1.67 to 2.22). Among those with diabetes, there was no significant difference between the sexes in achieving the HbA1c targets ( $41 \%$ of women vs $43 \%$ of men). In contrast, a smaller percentage of women than men reached the treatment targets for TC $(8 \%$ vs $14 \%)$, LDL cholesterol ( $22 \%$ vs $33 \%)$, and glucose $(71 \%$ vs. $76 \%)$, respectively. After adjustment for age, women had 50\%, $43 \%, 22 \%$ lower odds than men of achieving TC, LDL cholesterol, and glucose targets (figure 1). Similarly, women had an $18 \%$ higher odds of being obese, a $26 \%$ lower odds of being physically active, and a $40 \%$ lower odds of attending cardiac rehabilitation than men. Overall, $6 \%$ of women and $8 \%$ of men reached all treatment targets and about one-third of men and women met all lifestyle targets; the ORs were 0.75 (95\% CI 0.60 to 0.93 ) for all treatment targets and 0.93 (95\% CI 0.84 to 1.02) for all lifestyle targets. Combined, $16 \%$ of women and $21 \%$ of men had an adequate $\mathrm{CHIS}$, which corresponded to a $19 \%$ lower odds in women than in men (OR $0.81,95 \%$ CI 0.73 to 0.91 ) (figure 2).

Findings were similar in the analyses adjusted for major risk factors (supplementary eFigure 1). Stratification of our analyses by medication use or CHD disease category did not materially change the results (supplementary eTable 2).

\section{Sex differences by region}

There was some indication that sex differences in the target achievements differed between regions (table 1 and figure 3). In Europe, the odds of achieving treatment targets for TC, LDL cholesterol, and glucose, respectively, were $34 \%, 31 \%$, and $14 \%$ lower in women than men, compared with a $70 \%, 47 \%$, and $30 \%$ lower odds for women in Asia, and a 76\%, 53\%, and $47 \%$ lower odds for achieving these targets for women in the Middle East. Sex differences in achieving all treatment targets were smallest in Europe and largest in the Middle East. Women in Asia and the Middle East were considerably more likely than men to be non-smokers, whereas no significant differences in smoking rates were observed between sexes in Europe (Asia: OR 11.5, 95\% CI 7.4 to 19.0; Middle East: OR 16.2, 95\% CI 6.7 to 53.4; Europe: OR 1.1, 95\% CI 0.9 to 1.3). In Asia, women were more likely to be physically active than men. In contrast, women in Europe and the Middle East were less physically active than their male counterparts (figure 3). In Asia, women were more likely than men to meet all lifestyle targets, but the reverse was seen in Europe and the Middle East.

In Asia, the odds of having an adequate CHIS was $33 \%$ higher in women than men, compared with a $29 \%$ and $49 \%$ lower odds in women than men in Europe and the Middle East, respectively.

\section{Sex differences by age}

The sex differences in achieving treatment targets differed between those aged $\leq 65$ years and those $>65$ years for TC, LDL cholesterol, and glucose, but not for other risk factors (figure 4). Compared with younger men, younger women were $59 \%$ less likely to meet the TC target, 53\% less likely to meet the LDL cholesterol target, and $28 \%$ less likely to meet the glucose target. Corresponding results in those aged $>65$ years were $42 \%, 34 \%$, and $18 \%$, respectively. There was no evidence that women's lower odds of meeting all treatment targets, all lifestyle targets, or having an adequate CHIS, compared with men, differed between those aged $\leq 65$ versus $>65$ years.

\section{Sex differences by age and region}

Regional differences in achieving treatment and lifestyle targets varied between younger and older individuals 


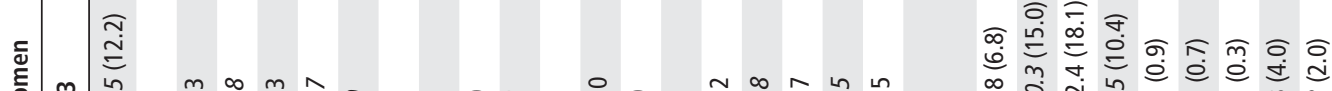

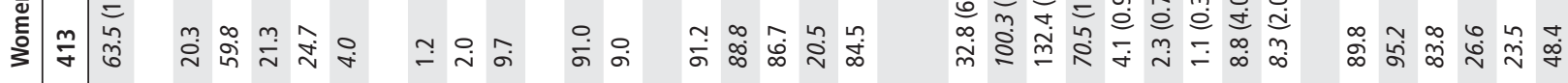

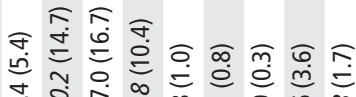

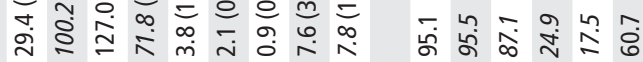

لla

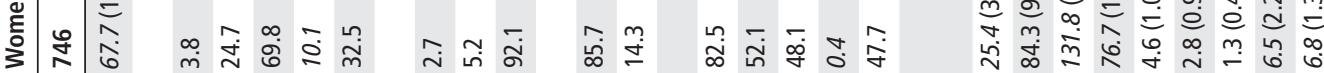

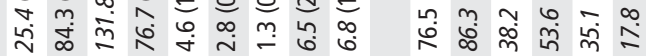

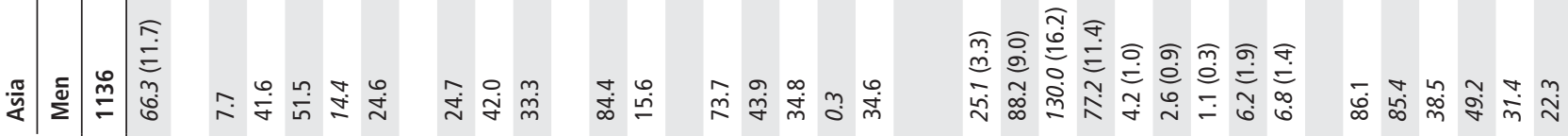

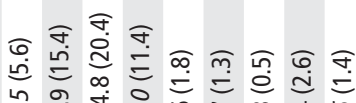

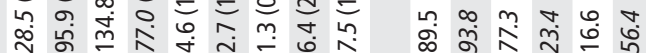

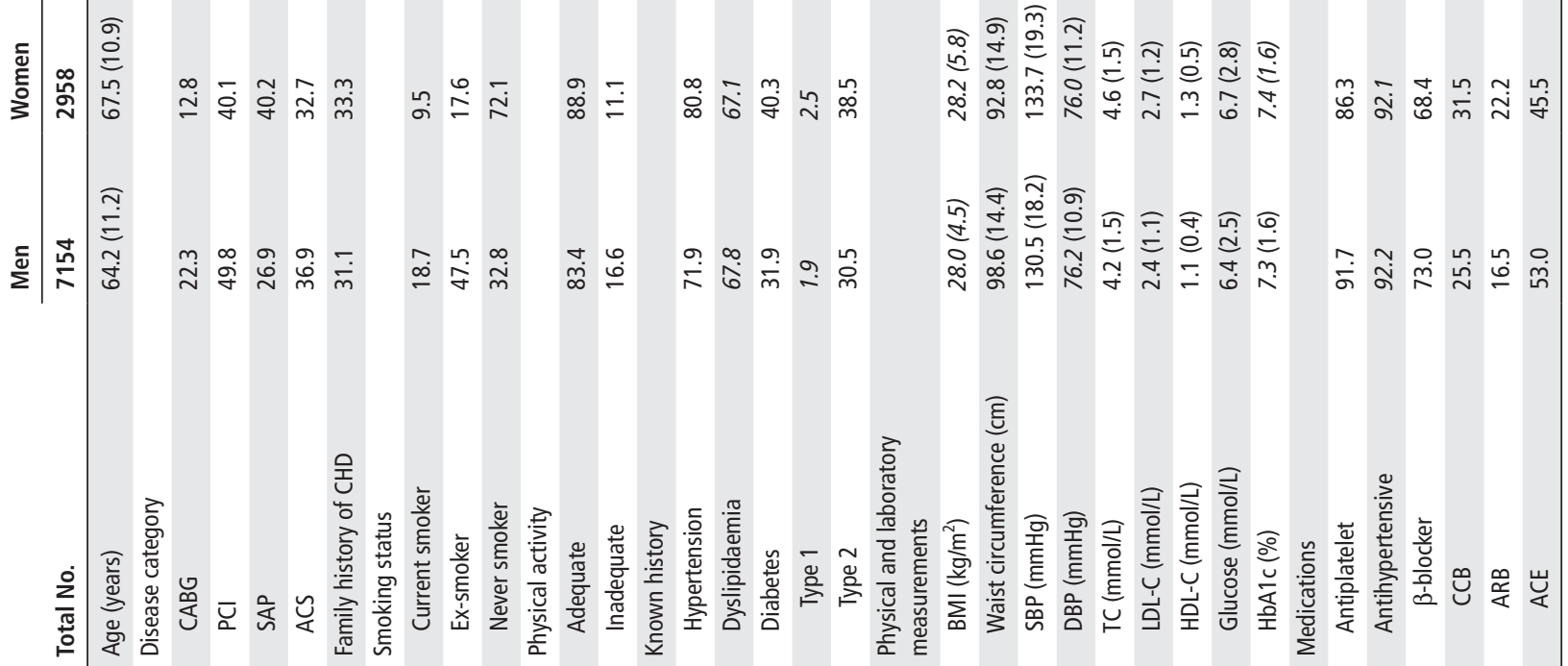

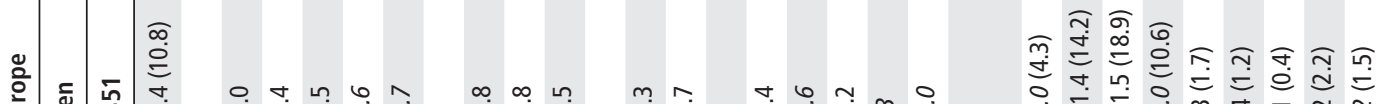

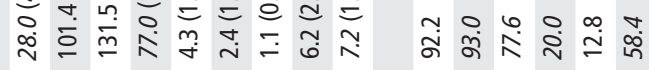
ป में 


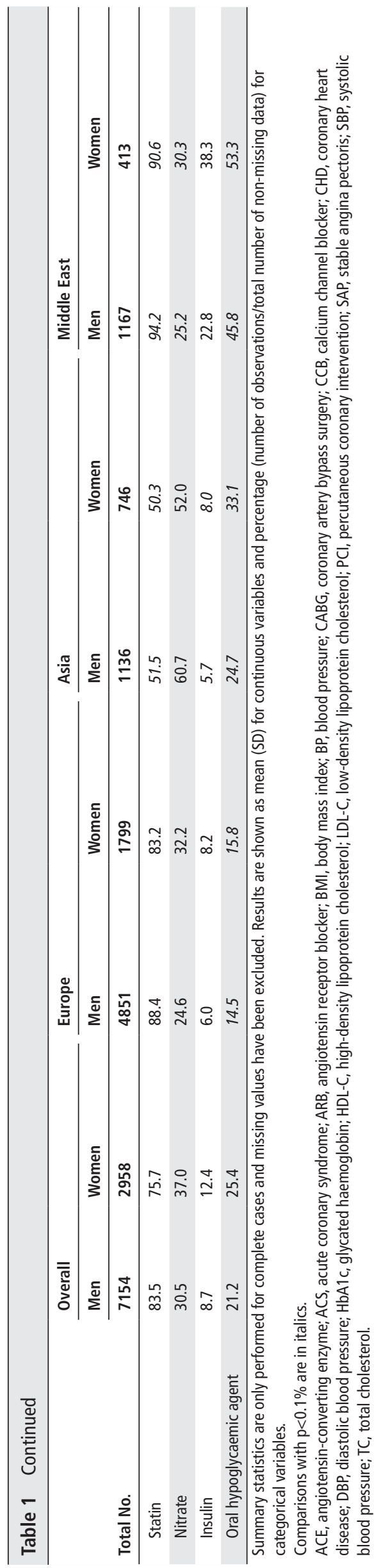

(supplementary eTable 3). Due to higher smoking prevalence in younger men, in Asia and the Middle East, sex differences in smoking rates tended to be larger in those aged $\leq 65$ years than in those aged $>65$ years in these regions. In Europe and the Middle East, sex differences in overall risk factor management, to women's disadvantage, were larger among younger than among older individuals. In Asia, the odds of adequate overall risk factor management (ie, a good CHIS) was higher among younger women than younger men, which was largely driven by better control of lifestyle factors in women. In older women in Asia, the odds of adequate risk factor management was lower than in their male counterparts.

\section{DISCUSSION}

The present study among over 10000 individuals with CHD indicated that, overall, risk factor management for the secondary prevention of CHD is worse among women than men. However, the magnitude and direction of the sex differences in the likelihood of meeting guideline-recommended targets varied across component treatment and lifestyle targets. BP control was better in women than in men whereas women were less likely to reach target lipid and glucose levels. Sex differences in risk factor management also varied across regions, with contrasting patterns for treatment and lifestyle targets.

Previous studies on sex differences in risk factor management for the secondary prevention of CHD have also shown that women, in general, have a worse risk factor profile and are less likely to meet therapeutic targets than men. The EUROASPIRE III and IV, two large surveys on the control of cardiovascular risk factors among coronary patients across Europe, reported that women were less likely than men to achieve target lipid and HbA1c levels. ${ }^{67}$ Additionally, EUROASPIRE IV demonstrated that the largest sex differences were seen among elderly patients and among those with lower levels of education. ${ }^{6}$ EUROASPIRE III reported that BP control was also worse among women than men, whereas EUROASPIRE IV found similar rates between sexes. ${ }^{67}$ In the present study, we found that, although BP levels were higher among women than men, BP control was considerably better in women than in men. Lipid and glucose targets, however, were less likely to be achieved by women than men.

Sex differences in the availability of evidence-based medications may be responsible for women's lower likelihood of achieving treatment targets. While the EUROASPIRE surveys reported broadly similar treatment rates between sexes, ${ }^{67}$ our results are consistent with several other studies indicating lower rates of medication use in women than men. ${ }^{12-16}$ For instance, the CRUSADE study, a large national study involving 36000 coronary patients in the USA, demonstrated that women were less likely than men to receive aspirin, ACE inhibitors, or statins at hospital discharge after a cardiac event, even after adjustment for women's worse cardiovascular risk profile at admission. ${ }^{17}$ Moreover, a study among 15000 coronary patients in the Netherlands found persistent sex differences in the use of lipid-lowering and antithrombotic medications, particularly in younger patients. ${ }^{13}$ Others also reported that women were less likely than men to receive intensive lipid-lowering therapy in order to achieve their optimal lipid goals. ${ }^{14}{ }^{16}$ However, our analyses stratified by medication use did not alter our main findings on sex differences of risk factor management; nevertheless, the prevalence of medication use differs between sexes.

Despite this, as shown in our findings that women with CHD tended to be older, women's older age at the diagnosis of CHD with more comorbidities might also explain their lower 


$\begin{array}{ll}\text { Treatment target } & \\ \text { BP on target } & 45 \\ \text { TC on target } & 8 \\ \text { LDL on target } & 22 \\ \text { HDL on target } & 51 \\ \text { Glucose on target } & 71 \\ \text { HbA1c on target } & 41 \\ \text { All treatment targets } & 6 \\ & \\ \text { Lifestyle } & \\ \text { Non-smoker } & 90 \\ \text { Adequate physical activites } & 48 \\ \text { Non-obese } & 69 \\ \text { Non-central obese } & 32 \\ \text { Cardiac rehabilitation } & 23 \\ \text { All lifestyle targets } & 32\end{array}$

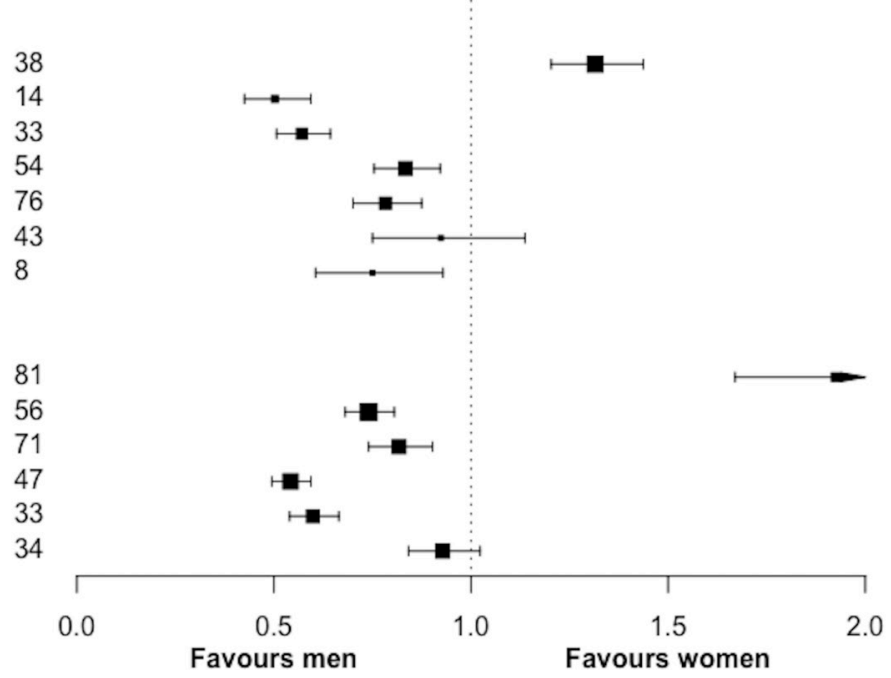

$1.31[1.20,1.44]$

$0.50[0.43,0.59]$

$0.57[0.51,0.64]$

$0.83[0.75,0.92]$

$0.78[0.70,0.87]$

$0.92[0.75,1.14]$

$0.75[0.60,0.93]$

$1.93[1.67,2.22]$

$0.74[0.68,0.81]$

$0.82[0.74,0.90]$

$0.54[0.49,0.59]$

$0.60[0.54,0.66]$

$0.93[0.84,1.02]$

Favours men

Favours women

Figure 1 Age-adjusted sex differences in risk factor management. Target blood pressure (BP) was defined as BP $<140 / 90 \mathrm{mmHg}$ in those without diabetes or $<140 / 80 \mathrm{mmHg}$ in those with diabetes. The target for total cholesterol (TC), low-density lipoprotein (LDL) cholesterol and high-density lipoprotein (HDL) cholesterol levels were defined as $<3 \mathrm{mmol} / \mathrm{L},<1.8 \mathrm{mmol} / \mathrm{L}$, and $>1.0 \mathrm{mmol} / \mathrm{L}$ for men and $>1.2 \mathrm{mmol} / \mathrm{L}$ for women, respectively. Target glucose was defined as $<7 \mathrm{mmol} / \mathrm{L}$. Information on glycated haemoglobin (HbA1c) was only collected from patients with diabetes and its target was defined as $<7 \%$. Achieving all three medical targets (BP on target, LDL on target, and glucose/HbA1c on target) was defined as 'All treatment targets'. Obesity was defined as a body mass index (BMI) $\geq 30 \mathrm{~kg} / \mathrm{m}^{2}$ and central obesity was defined as waist circumference $\geq 88 \mathrm{~cm}$ for women and $\geq 102 \mathrm{~cm}$ for men. Smoking status was current smoker and non-smoker. Adequate physical activity level was defined as moderate or vigorous physical activity for at least 30 min three or more times a week. Reaching all three lifestyle targets (non-smoker, adequate physical activities, and nonobesity) was defined as 'All lifestyle targets'. Odds ratios $(95 \% \mathrm{Cl})$ presented as women versus men.

likelihood of receiving pharmacological therapy. ${ }^{18} 19$ This is also problematic for younger women when their CHD conditions are often considered less serious, compared with men. ${ }^{18}$ Consistent with previous evidence, ${ }^{13}$ the sex differences in achieving lipid targets in this study were larger among younger than among older patients, indicating that younger women are particularly disadvantaged. Furthermore, our findings revealed differential distribution of CHD category between women and
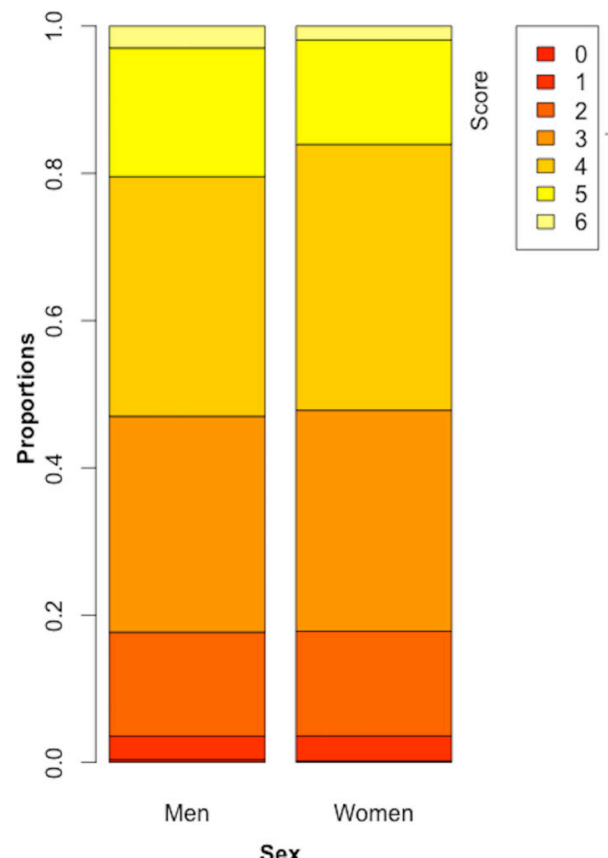

Subgroup Women(\%) Men(\%)

Odds ratio[95\% $\mathrm{Cl}] \quad \mathrm{P}$ value

Region

EU $\quad 18 \quad 23$

Asia $\quad 18 \quad 17$

Middle East

313

3

$\begin{array}{lll}\text { Age } & & \\ \leq 65 & 14 & 18 \\ >65 & 18 & 23\end{array}$

$0.84[0.71,0.99]$

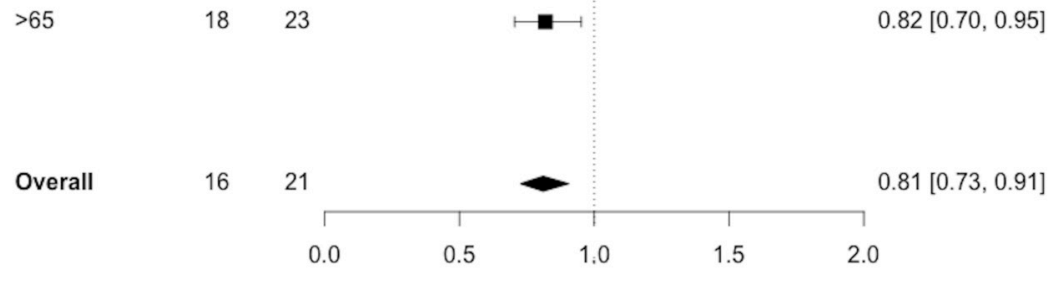

Favours men

Favours women

Figure 2 Age-adjusted sex differences in Cardiovascular Health Index Score (CHIS). The CHIS included six risk factors: smoking status (current smoker or non-smoker), body mass index (obese or not), physical activity (adequate or not), blood pressure (on target or not), low-density lipoprotein (LDL) cholesterol (on target or not), and $\mathrm{HbA1}$ c/glucose (on target or not). The number of controlled risk factors was summed, ranging from 0 to 6 . A good CHIS was defined as five or more risk factors controlled (CHIS $=5$ or 6$)$. Odds ratios $(95 \% \mathrm{Cl})$ presented as women versus men; $p$ values are for interaction between subgroups. 


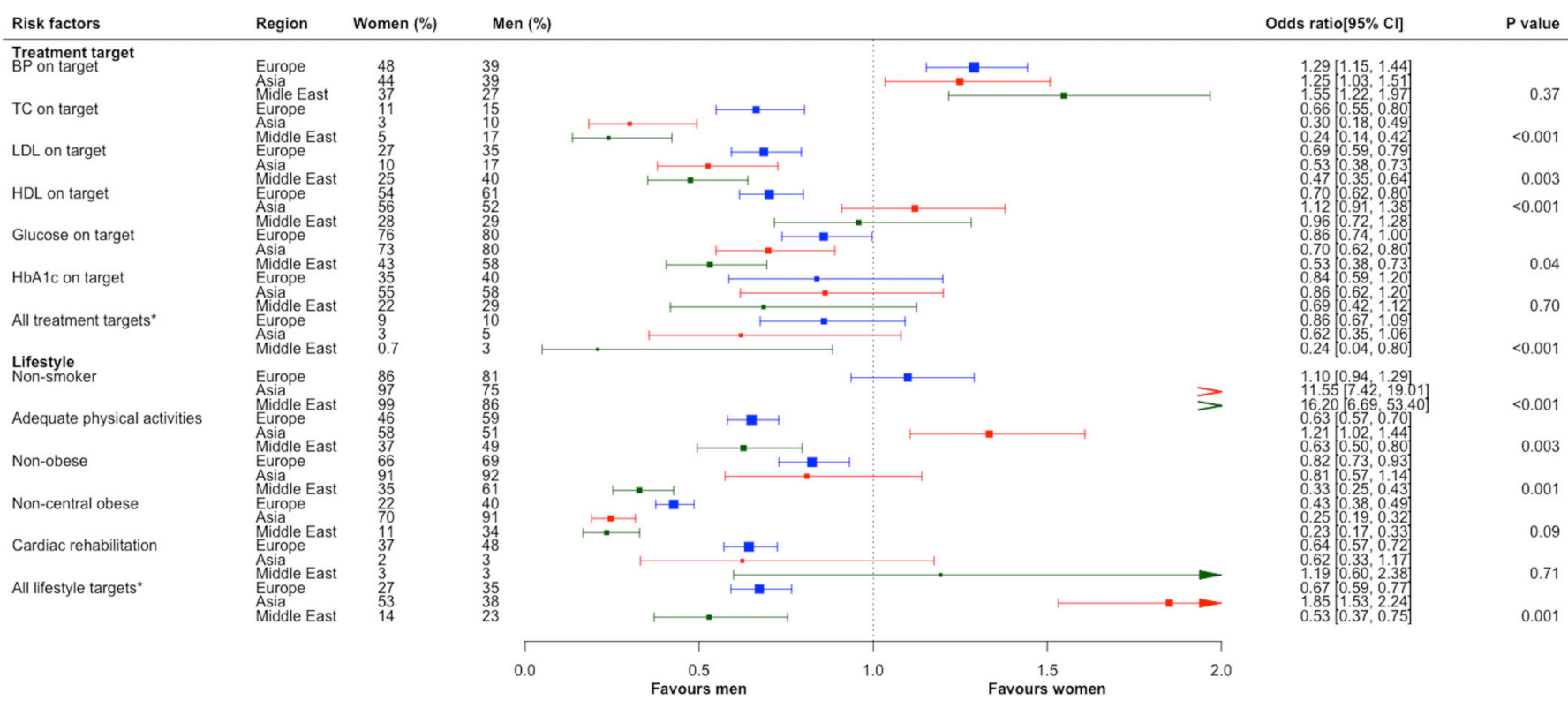

Figure 3 Age-adjusted sex differences on treatment targets and lifestyle factor management, stratified by region. Conventions as in figure 1. Odds ratios $(95 \% \mathrm{Cl})$ presented as women versus men; $p$ values are for interaction between subgroups.

men and fewer female CABG patients were recruited. As such, women may pay less attention to their CHD risk factor management, resulting in less cardiovascular medication being used and fewer targets being achieved by women. This is unfortunate as clinical guidelines recommend, based on evidence from large randomised controlled trials, the use of preventative medications and a strategy of CHD prevention for all CHD patients, irrespective of age, sex, or severity of disease. ${ }^{20}$

Most previous studies on sex differences in cardiovascular risk management have been conducted in Western populations. Our study not only showed that substantial sex differences in cardiovascular risk management exist in Europe, Asia and the Middle East, but also indicated that regional variations in the size and direction of these sex disparities are present. Sex differences in smoking habits varied most notably across regions; while the prevalence of smoking was similar between the sexes in Europe, women in Asia and the Middle East were considerably less likely to smoke than their male counterparts. In contrast, sex disparities in the achievement of treatment targets were smaller in Europe than in Asia and the Middle East, especially for lipid and glucose levels. Lack of knowledge among female patients about their disease or the necessity of adequate guideline-recommended treatment could contribute to these sex differences in risk factor management. ${ }^{19}{ }^{21-24} \mathrm{~A}$ survey in the USA found that only $55 \%$ of women were aware that CHD is the leading cause of death in women and less than half of the women were

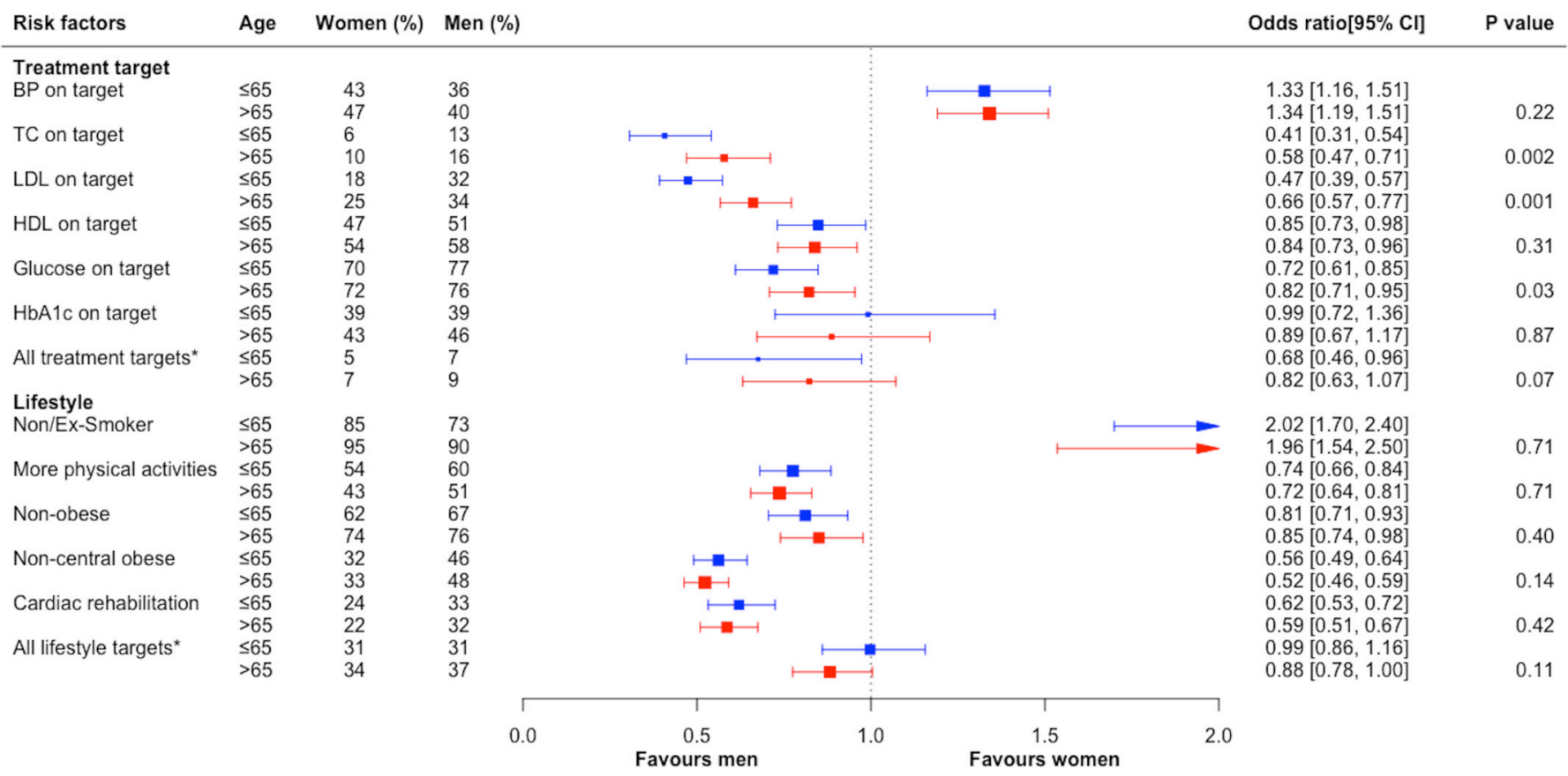

Figure 4 Sex differences in treatment targets and lifestyle factor management, stratified by age. Conventions as in figure 1.0 dds ratios (95\% $\mathrm{Cl}$ ) presented as women versus men; $p$ values are for interaction between subgroups. 
familiar with optimal levels of CHD risk factors. ${ }^{22}$ Additionally, a 12-year follow-up survey in the USA showed that the majority of women did not adhere to appropriate secondary prevention and often used non-evidence-based therapies to prevent CHD. ${ }^{23}$ Comparative studies among men have not been conducted, and neither are there robust data on the awareness of CHD risk among women, and men, from non-Western populations. However, it is conceivable that women's awareness of CHD risk and the benefits of the management of major risk factors is particularly low in non-Western populations, where CHD rates are increasingly rising, risk factor profiles are different, and the uptake of preventive strategies remains lower than in the West. Greater knowledge and awareness of CHD in women, better understanding of regional differences, as well as more widespread use of women-specific clinical guidelines appropriate to local settings, could help to decrease the sex disparities in CHD risk factor management and could improve CHD outcomes in both men and women.

SURF, a pragmatic clinical audit, is undertaken as part of routine clinics at low cost and minimal increase in workload, aiming to document and investigate CHD risk factor management for secondary prevention. It is particularly suitable as an audit instrument for use in low-resource settings and facilitates multiple comparisons of risk factor management across different regions and, in future iterations, over time. Some limitations of SURF deserve to be mentioned. Unlike EUROASPIRE, SURF data are collected during outpatient visits and laboratory measurements are not performed with a standardised scientific methodology. Although the high frequency of missing data might reduce the reliability of prevalence estimates, the percentage of missing data within each region was broadly similar between sexes, and hence it is unlikely to alter our conclusions. Moreover, participating centres were identified through personal contact and may not be representative of healthcare facilities treating CHD patients in participating countries. Finally, more than $60 \%$ of patients were recruited from European centres. While these limitations may have affected the descriptive characteristics, the comparisons of sex differences in cardiovascular risk factor management are less likely to be affected. A new phase of SURF, SURF II, will increase representativeness both in terms of patient participation and by allowing the participation of a wide variety of centres, irrespective of size and resources.

\section{Key messages}

What is already known about this subject?

Prior studies in mostly Western populations have suggested a sex disparity in the management of cardiovascular risk factors among patients with established coronary heart disease (CHD).

What does this study add?

This study assessed sex differences in risk factor management for the secondary prevention of CHD across three geographically diverse regions; Europe, Asia, and the Middle East. These data show that sex disparities in reaching treatment targets in Asia and the Middle East were greater than in Europe, to the detriment of women.

How might this impact on clinical practice? A better understanding of sex disparities in CHD is needed to treat women with CHD more efficiently in all regions, especially in Asia and the Middle East.

\section{CONCLUSION}

We observed substantial differences between men and women in cardiovascular risk factor management for the secondary prevention of CHD, most often to the detriment of women. Sex disparities in risk factor management differed across regions, suggesting the need for tailored strategies to reduce these inequalities and to improve the uptake of guideline-recommended care for the secondary prevention of $\mathrm{CHD}$ in both men and women.

\section{Author affiliations}

Julius Global Health, Julius Center for Health Sciences and Primary Care, University Medical Center Utrecht, Utrecht, The Netherlands

${ }^{2}$ Julius Center for Health Sciences and Primary Care, University Medical Center Utrecht, Utrecht, The Netherlands

${ }^{3}$ Global Geo and Health Data Center, Utrecht University, Utrecht, The Netherlands

${ }^{4}$ Trinity College Dublin, Dublin, Ireland

${ }^{5}$ Department of Vascular Medicine, University Medical Center Utrecht, Utrecht, The Netherlands

${ }^{6}$ Division of Epidemiology \& Biostatistics, School of Public Health, Faculty of Health Sciences, University of the Witwatersrand, Johannesburg, South Africa

${ }^{7}$ The George Institute for Global Health, University of Oxford, Oxford, United Kingdom

${ }^{8}$ The George Institute for Global Health, University of New South Wales, Sydney, NSW, Australia

Acknowledgements The SURF study group is grateful to all participating centres and their staff for their participation and support.

Contributors MZ, SP, MW, KK, IV, RG, and IG conceived and designed the study. $M Z, S P$, and $M W$ analyzed and interpreted the data. MZ drafted the manuscript and all authors contributed to critical revision of the manuscript.

Funding $\mathrm{MZ}$ is supported by a grant from the Netherlands Organization for Scientific Research (NWO; grant number: 0.22.005.021). NWO had no input to the design, execution, analysis, or writing up of the study. IV is supported by a grant from the Dutch Heart Foundation (grant DHF project 'Facts and Figures'). MW is supported by the National Health and Medical Research Foundation of Australia (1080206). SP is supported by the British Heart Foundation (PG/16/57/32256)

Competing interests MW is a consultant to Amgen on analyses of Medicare data in the USA. Amgen had no input to the design, execution, analysis, or writing up of the study

Provenance and peer review Not commissioned; externally peer reviewed.

Open Access This is an Open Access article distributed in accordance with the Creative Commons Attribution Non Commercial (CC BY-NC 4.0) license, which permits others to distribute, remix, adapt, build upon this work non-commercially, and license their derivative works on different terms, provided the original work is properly cited and the use is non-commercial. See: http://creativecommons.org/ licenses/by-nc/4.0/

(c) Article author(s) (or their employer(s) unless otherwise stated in the text of the article) 2017. All rights reserved. No commercial use is permitted unless otherwise expressly granted.

\section{REFERENCES}

1 GBD 2015 Mortality and Causes of Death Collaborators. Global, regional, and national life expectancy, all-cause mortality, and cause-specific mortality for 249 causes of death, 1980-2015: a systematic analysis for the Global Burden of Disease Study 2015. Lancet 2016;388:1459-544.

2 Piepoli MF, Hoes AW, Agewall S, et al. Authors/Task Force Members:. 2016 European Guidelines on cardiovascular disease prevention in clinical practice: The Sixth Joint Task Force of the European Society of Cardiology and Other Societies on Cardiovascular Disease Prevention in Clinical Practice (constituted by representatives of 10 societies and by invited experts) Developed with the special contribution of the European Association for Cardiovascular Prevention \& Rehabilitation (EACPR) Atherosclerosis 2016;252:207-74.

3 Kotseva K, Wood D, De Bacquer D, et al. EUROASPIRE Investigators. EUROASPIRE IV: A European Society of Cardiology survey on the lifestyle, risk factor and therapeutic management of coronary patients from 24 European countries. Eur J Prev Cardiol 2016;23:636-48

4 Zhao M, Cooney MT, Klipstein-Grobusch K, et al. Simplifying the audit of risk factor recording and control: a report from an international study in 11 countries. Eur J Prev Cardiol 2016;23:1202-10.

5 Cooney M, Reiner Z, Sheu W, et al. SURF - SUrvey of Risk Factor management: first report of an international audit. Eur J Prev Cardiol 2014;21:813-22. 
6 De Smedt D, De Bacquer D, De Sutter J, et al. The gender gap in risk factor control: effects of age and education on the control of cardiovascular risk factors in male and female coronary patients. The EUROASPIRE IV study by the European Society of Cardiology. Int J Cardiol 2016;209:284-90.

7 Dallongevillle J, De Bacquer D, Heidrich J, et al. Gender differences in the implementation of cardiovascular prevention measures after an acute coronary event. Heart 2010;96:1744-9.

8 Mosca L, Barrett-Connor E, Wenger NK, Kass Wenger N. Sex/gender differences in cardiovascular disease prevention: what a difference a decade makes. Circulation 2011; 124:2145-54.

9 Perk J, De Backer G, Gohlke H, et al. European Guidelines on cardiovascular disease prevention in clinical practice (version 2012). Eur Heart J 2012;33:1635-701.

10 WHO Expert Consultation. Appropriate body-mass index for Asian populations and its implications for policy and intervention strategies. Lancet 2004;363:157-63.

11 Lloyd-Jones DM, Hong Y, Labarthe D, et al. Defining and setting national goals for cardiovascular health promotion and disease reduction: the American Heart Association's strategic impact goal through 2020 and beyond. Circulation 2010;121:586-613.

12 Simpson CR, Hannaford PC, Williams D. Evidence for inequalities in the management of coronary heart disease in Scotland. Heart 2005;91:630-4.

13 Koopman C, Vaartjes I, Heintjes EM, et al. Persisting gender differences and attenuating age differences in cardiovascular drug use for prevention and treatment of coronary heart disease, 1998-2010. Eur Heart J 2013;34:3198-205.

14 Victor BM, Teal V, Ahedor L, et al. Gender differences in achieving optimal lipid goals in patients with coronary artery disease. Am J Cardiol 2014;113:1611-5.

15 DeWilde S, Carey IM, Richards N, et al. Trends in secondary prevention of ischaemic heart disease in the UK 1994 2005: use of individual and combination treatment. Heart 2008;94:83-8.
16 Virani SS, Woodard LD, Ramsey DJ, et al. Gender disparities in evidence-based statin therapy in patients with cardiovascular disease. Am J Cardiol 2015;115:21-6.

17 Blomkalns AL, Chen AY, Hochman JS, et al. Gender disparities in the diagnosis and treatment of non-ST-segment elevation acute coronary syndromes: large-scale observations from the CRUSADE (Can Rapid Risk Stratification of Unstable Angina Patients Suppress Adverse Outcomes with Early Implementation of the American College of Cardiology/American Heart Association guidelines) National Quality Improvement Initiative. J Am Coll Cardiol 2005:45:832-7.

18 Zhang H, Plutzky J, Shubina M, et al. Drivers of the sex disparity in statin therapy in patients with coronary artery disease: a cohort study. PLoS One 2016;11:e0155228.

19 Maas AH, Van Der Schouw YT, Regitz-Zagrosek V, et al. Red alert for women's heart: the urgent need for more research and knowledge on cardiovascular disease in women. Eur Heart J 2011;32:1362-8.

20 O'Gara PT, Kushner FG, Ascheim DD, et al. American College of Emergency PhysiciansSociety for Cardiovascular Angiography and Interventions. 2013 ACCF/AHA guideline for the management of ST-elevation myocardial infarction: a report of the American College of Cardiology Foundation/American Heart Association Task Force on Practice Guidelines. J Am Coll Cardiol 2013;61:e78-140.

21 Shah T, Palaskas N, Ahmed A. An Update on gender disparities in coronary heart disease care. Curr Atheroscler Rep 2016;18:28.

22 Ramachandran HJ, Wu VX, Kowitlawakul Y, et al. Awareness, knowledge and healthy lifestyle behaviors related to coronary heart disease among women: an integrative review. Heart Lung 2016;45:173-85.

23 Mosca L, Mochari-Greenberger H, Dolor RJ, et al. Twelve-year follow-up of American women's awareness of cardiovascular disease risk and barriers to heart health. Circ Cardiovasc Qual Outcomes 2010;3:120-7.

24 Mosca L, Mochari H, Christian A, et al. National study of women's awareness, preventive action, and barriers to cardiovascular health. Circulation 2006;113:525-34. 